\title{
Electromagnetic response in kinetic energy driven cuprate superconductors: Linear response approach
}

\author{
Mateusz Krzyzosiak \\ Department of Physics, Beijing Normal University, Beijing 100875, China and \\ Institute of Physics, Wroctaw University of Technology, \\ Wybrzeże Wyspiańskiego 27, 50-370 Wroctaw, Poland \\ Zheyu Huang and Shiping Feng* \\ Department of Physics, Beijing Normal University, Beijing 100875, China \\ Ryszard Gonczarek \\ Institute of Physics, Wroctaw University of Technology, \\ Wybrzeże Wyspiańskiego 27, 50-370 Wroctaw, Poland
}

\begin{abstract}
Within the framework of the kinetic energy driven superconductivity, the electromagnetic response in cuprate superconductors is studied in the linear response approach. The kernel of the response function is evaluated and employed to calculate the local magnetic field profile, the magnetic field penetration depth, and the superfluid density, based on the specular reflection model for a purely transverse vector potential. It is shown that the low temperature magnetic field profile follows an exponential decay at the surface, while the magnetic field penetration depth depends linearly on temperature, except for the strong deviation from the linear characteristics at extremely low temperatures. The superfluid density is found to decrease linearly with decreasing doping concentration in the underdoped regime. The problem of gauge invariance is addressed and an approximation for the dressed current vertex, which does not violate local charge conservation is proposed and discussed.
\end{abstract}

PACS numbers: 74.25.Ha, 74.25.Nf, 74.20.Mn

Keywords: Electromagnetic response; Magnetic field penetration depth; Cuprate superconductors

\section{INTRODUCTION}

Observation of superconductor's response to a weak external electromagnetic stimulus allows us to collect a number of subtle characteristics ${ }^{2}$. The way the magnetic field is expelled from a superconducting (SC) sample in the spectacular Meissner effect can be used to infer about many fundamental features of the system. Therefore the phenomena at the length scale of the magnetic field penetration depth $\lambda$, i.e. in the region at the edge of the sample where the induced supercurrents effectively screen the external magnetic field, are subject to intensive studies both on the theoretical and the experimental fronts of the research in cuprate superconductors 3.4 . In particular, the magnetic field penetration depth can be used as a probe of the pairing symmetry since it can distinguish between a fully gapped and a nodal quasiparticle excitation spectrum ${ }^{3,4}$. The former results in the thermally activated (exponential) temperature dependence of the magnetic field penetration depth, whereas the latter one implies a power law behavior.

The magnetic field penetration depth is a basic parameter of superconductors, closely related to the superfluid density ${ }^{2}$. Earlier on, the linear temperature dependence of the magnetic field penetration depth $\lambda(T)$ was observed for the cuprate superconductor $\mathrm{YBa}_{2} \mathrm{Cu}_{3} \mathrm{O}_{7-y}$ at low temperatures $(T=4 \mathrm{~K} \sim 20 \mathrm{~K})^{\frac{5}{5}}$, which first provided a strong experimental support for the nodes in the d-wave SC gap function of cuprate superconduc- tors, then confirmed by the angle-resolved photoemission spectroscopy (ARPES) experiments $\frac{6.7}{\text {. }}$. Later, this linear temperature dependence of the magnetic field penetration depth has been observed in different families of cuprate superconductors $\stackrel{8-11}{-1}$. However, at extremely low temperatures $(T<4 \mathrm{~K})$, the linear temperature dependence of the magnetic field penetration depth is modified, and a nonlinearity emerges ${ }^{12-14}$. Moreover, some indications of nonlocal effects giving rise to the nonlinearity have been reported in the field dependence of the effective magnetic field penetration depth in cuprate superconductors ${ }^{14}$. Furthermore, the doping dependence of the electromagnetic response in cuprate superconductors has been studied in terms of the zero-temperature superfluid density. The superfluid density is proportional to the squared amplitude of the coherent macroscopic wave function describing the SC charge carriers, and therefore it is an important physical quantity and can provide significant information about the SC state. In particular, the superfluid density of cuprate superconductors in the underdoped regime vanishes more or less linearly with decreasing doping concentration $15-17$. This in turn gives rise to the linear relation between the critical temperature $T_{\mathrm{c}}$ and the superfluid density observed in the underdoped regime $\frac{15}{}$.

Theoretically, the electromagnetic response in cuprate superconductors has been extensively studied based on the the phenomenological Bardeen-Cooper-Schrieffer (BCS) formalism with the d-wave SC gap function ${ }^{18-22}$. 
It has been shown ${ }^{4,19}$ that for a d-wave superconductor in the local limit $(\zeta \ll \lambda$, where $\zeta$ is the coherence length), the simple d-wave pairing state (assuming a tetragonal symmetry and ignoring the dispersion in the caxis direction) gives the magnetic field penetration depth $\lambda(T) \propto T / \Delta_{0}$, where $\Delta_{0}$ is the zero-temperature value of the d-wave gap amplitude. In particular, it has been argued that this linear temperature dependence of the magnetic field penetration depth is attributed to the excitation of quasiparticles out of the condensate at the nodes of the SC gap function. Furthermore, the fact $\mathrm{B}^{-11}$ that this linear relation holds down to very low doping concentrations suggests that near the nodes these quasiparticle excitations are well described by a simple BCSlike formalism with the d-wave SC gap function, even for the doping concentration $\delta \rightarrow 022$. This is also consistent with the ARPES experiments 23 . However, this depends sensitively on the quasiparticle scattering. In particular, at extremely low temperatures, the coherence length may diverge at the nodes. This may imply that the local condition no longer holds, and the electromagnetic field varies significantly over the size of a Cooper pair. Consequently, the nonlocal effect emerges 13 and then plays an important role in the electromagnetic response of cuprate superconductors ${ }^{18-22}$. It has been suggested ${ }^{18-22}$ that nonlocal effects can imply a crossover from the linear temperature dependence of the magnetic field penetration depth at low temperatures to a nonlinear one in the extremely low temperature range. To the best of our knowledge, the electromagnetic response in cuprate superconductors has not been treated starting from a microscopic SC theory, and no explicit calculations of the doping dependence of the superfluid density in the underdoped regime have been made so far.

Recently, a kinetic energy driven SC mechanism has been developed 24 , where the charge carrier-spin interaction from the kinetic energy term induces a charge carrier pairing state with the d-wave symmetry by exchanging spin excitations. Then the electron Cooper pairs originating from the charge carrier pairing state are due to charge-spin recombination, and their condensation reveals the d-wave SC ground-state. In particular, this SCstate is controlled by both the SC gap function and the quasiparticle coherence, then the maximal SC transition temperature occurs around the optimal doping, and decreases in both underdoped and overdoped regimes. The unique feature of this kinetic energy driven SC mechanism is that the pairing comes out from the kinetic energy by exchanging spin excitations and is not driven by the magnetic superexchange interaction as in the resonant valence bond type theories ${ }^{25}$. Within the framework of the kinetic energy driven superconductivity, we have discussed the low energy electronic structure $\frac{26,27}{27}$ of cuprate superconductors and the spin response $\mathrm{e}^{24.28}$, and qualitatively reproduced some main features of ARPES experiments 6.7 and inelastic neutron scattering 29,30 measurements on cuprate superconductors.

The layered crystal structure gives rise to a strong anisotropy of cuprate superconductors, and it is possible to observe both in-plane and inter-plane electromagnetic responses. The former one is characterized by the abplane magnetic field penetration depth, whereas the latter one is related to the magnetic field penetration in the c-axis direction. In this paper we concentrate on the inplane electromagnetic response based on the kinetic energy driven superconductivity and do not consider c-axis properties, which can be discussed, e.g., by taking into account hopping between adjacent copper-oxides layers within the tunneling Hamiltonian approach ${ }^{22}$.

The paper is organized as follows. Within the framework of the kinetic energy driven d-wave superconductivity $\stackrel{24}{ }$, we discuss the electromagnetic response of cuprate superconductors in Section [II] deriving the kernel of the linear response with a purely transverse vector potential. In Section III based on the specular reflection mode ${ }^{31.32}$, we calculate the temperature and doping dependence of quantitative characteristics of the electromagnetic response, such as the local magnetic field profile, the magnetic field penetration depth, and the superfluid density. Our results show that the electromagnetic response in cuprate superconductors can be understood within the framework of the kinetic energy driven d-wave SC mechanism in the presence of a weak external magnetic field. We conclude the paper with a brief summary in Section IV In Appendix @ we present a method to generalize the approach in order to obtain gauge invariant results.

\section{ELECTROMAGNETIC RESPONSE IN CUPRATE SUPERCONDUCTORS}

A common feature of cuprate superconductors is the presence of two-dimensional $\mathrm{CuO}_{2}$ planes, and it is believed that the unconventional physical properties of these systems are closely related to the doped $\mathrm{CuO}_{2}$ plane ${ }^{7}$. It has been argued that the essential physics of the doped $\mathrm{CuO}_{2}$ plane $\frac{7,25}{}$ is captured by the $t-J$ model on a square lattice. However, for discussions of the electromagnetic response in cuprate superconductors, the $t$ $J$ model can be extended by including the exponential Peierls factors as,

$$
\begin{aligned}
H & =-t \sum_{l \hat{\eta} \sigma} e^{-i(e / \hbar) \mathbf{A}(l) \cdot \hat{\eta}} C_{l \sigma}^{\dagger} C_{l+\hat{\eta} \sigma}+\mu \sum_{l \sigma} C_{l \sigma}^{\dagger} C_{l \sigma} \\
& +J \sum_{l \hat{\eta}} \mathbf{S}_{l} \cdot \mathbf{S}_{l+\hat{\eta}},
\end{aligned}
$$

where $\hat{\eta}= \pm \hat{x}, \pm \hat{y}, C_{l \sigma}^{\dagger}\left(C_{l \sigma}\right)$ is the electron creation (annihilation) operator, $\mathbf{S}_{l}=\left(S_{l}^{x}, S_{l}^{y}, S_{l}^{z}\right)$ are spin operators, $\mu$ is the chemical potential, and the exponential Peierls factors account for the coupling of electrons to the weak external magnetic field in terms of the vector potential $\mathbf{A}(l)^{33,34}$. This $t-J$ model is subject to an important local constraint $\sum_{\sigma} C_{l \sigma}^{\dagger} C_{l \sigma} \leq 1$ in order to 
avoid the double occupancy. The strong electron correlation in the $t-J$ model manifests itself by this local constraint ${ }^{25}$, which can be treated properly in analytical calculations within the charge-spin separation (CSS) fermion-spin theory ${ }^{35}$, where the constrained electron operators are decoupled as $C_{l \uparrow}=h_{l \uparrow}^{\dagger} S_{l}^{-}$and $C_{l \downarrow}=h_{l \downarrow}^{\dagger} S_{l}^{+}$, with the spinful fermion operator $h_{l \sigma}=e^{-i \Phi_{l \sigma}} h_{l}$ representing the charge degree of freedom together with some effects of spin configuration rearrangements due to the presence of the doped hole itself (charge carrier), while the spin operator $S_{l}$ represents the spin degree of freedom. In particular, it has been shown that under the decoupling scheme, this CSS fermion-spin representation is a natural representation of the constrained electron defined in the Hilbert subspace without double electron occupancy 26 . The advantage of this CSS fermion-spin approach is that the electron single occupancy local constraint is satisfied in analytical calculations. Furthermore, these charge carriers and spins are gauge invariant, and in this sense, they are real and can be interpreted as the physical excitations 36 . In this CSS fermion-spin representation, the $t-J$ model (11) can be expressed as,

$$
\begin{aligned}
H & =t \sum_{l \hat{\eta}} e^{-i(e / \hbar) \mathbf{A}(l) \cdot \hat{\eta}}\left(h_{l+\hat{\eta} \uparrow}^{\dagger} h_{l \uparrow} S_{l}^{+} S_{l+\hat{\eta}}^{-}\right. \\
& \left.+h_{l+\hat{\eta} \downarrow}^{\dagger} h_{l \downarrow} S_{l}^{-} S_{l+\hat{\eta}}^{+}\right) \\
& -\mu \sum_{l \sigma} h_{l \sigma}^{\dagger} h_{l \sigma}+J_{\mathrm{eff}} \sum_{l \hat{\eta}} \mathbf{S}_{l} \cdot \mathbf{S}_{l+\hat{\eta}},
\end{aligned}
$$

where $J_{\text {eff }}=(1-\delta)^{2} J$, and $\delta=\left\langle h_{l \sigma}^{\dagger} h_{l \sigma}\right\rangle=\left\langle h_{l}^{\dagger} h_{l}\right\rangle$ is the charge carrier doping concentration. As an important consequence, the kinetic energy term in the $t-J$ model has been transferred as the charge carrier-spin interaction, which reflects that even the kinetic energy term in the $t-J$ Hamiltonian has strong Coulomb contribution due to the restriction of no double occupancy of a given site.

In the case of zero magnetic field, we ${ }^{24}$ have shown in terms of Eliashberg's strong coupling theory ${ }^{37}$ that the charge carrier-spin interaction from the kinetic energy term in the $t-J$ model (2) induces a charge carrier pairing state with the d-wave symmetry by exchanging spin excitations in the higher power of the charge carrier doping concentration $\delta$, then the $\mathrm{SC}$ transition temperature is identical to the charge carrier pair transition temperature. Moreover, it has been shown that this SC state is the conventional BCS-like with the dwave symmetry 26,27 , so that the basic BCS formalism with the d-wave SC gap function is still valid in quantitatively reproducing all main low energy features of the $\mathrm{SC}$ coherence of the quasiparticle peaks in cuprate superconductors, although the pairing mechanism is driven by the kinetic energy by exchanging spin excitations, and other exotic magnetic scattering 29,30 is beyond the BCS formalism. Following the previous discussions $24,26,27$, the full charge carrier diagonal and off-diagonal Green's functions in the SC state can be obtained explicitly in the
Nambu representation as,

$$
\mathbb{G}\left(\mathbf{k}, i \omega_{n}\right)=Z_{\mathrm{hF}} \frac{i \omega_{n} \tau_{0}+\bar{\xi}_{\mathbf{k}} \tau_{3}-\bar{\Delta}_{\mathrm{hZ}}(\mathbf{k}) \tau_{1}}{\left(i \omega_{n}\right)^{2}-E_{\mathrm{hk}}^{2}},
$$

where $\tau_{0}$ is the unit matrix, $\tau_{1}$ and $\tau_{3}$ are Pauli matrices, the renormalized charge carrier excitation spectrum $\bar{\xi}_{\mathrm{k}}=Z_{\mathrm{hF}} \xi_{\mathbf{k}}$, with the mean-field (MF) charge carrier excitation spectrum $\xi_{\mathbf{k}}=Z t \chi \gamma_{\mathbf{k}}-\mu$, the spin correlation function $\chi=\left\langle S_{i}^{+} S_{i+\hat{\eta}}^{-}\right\rangle, \gamma_{\mathbf{k}}=(1 / Z) \sum_{\hat{\eta}} e^{i \mathbf{k} \cdot \hat{\eta}}$, $Z$ is the number of the nearest neighbor sites, the renormalized charge carrier d-wave pair gap function $\bar{\Delta}_{\mathrm{hZ}}(\mathbf{k})=Z_{\mathrm{hF}} \bar{\Delta}_{\mathrm{h}}(\mathbf{k})$, where the effective charge carrier d-wave pair gap function $\bar{\Delta}_{\mathrm{h}}(\mathbf{k})=\bar{\Delta}_{\mathrm{h}} \gamma_{\mathbf{k}}^{(d)}$ with $\gamma_{\mathbf{k}}^{(d)}=\left(\cos k_{x}-\cos k_{y}\right) / 2$, and the charge carrier quasiparticle spectrum $E_{\mathrm{hk}}=\sqrt{\bar{\xi}_{\mathbf{k}}^{2}+\left|\bar{\Delta}_{\mathrm{hZ}}(\mathbf{k})\right|^{2}}$, while the charge carrier quasiparticle coherent weight $Z_{\mathrm{hF}}$ and the effective charge carrier gap parameter $\bar{\Delta}_{\mathrm{h}}$ have been determined self-consistently along with another seven quantities and correlation functions $24,26,27$. Let us emphasize that the quasiparticle coherent weight renormalizing the physical quantities naturally emerges in our formalism (3), and then both the SC gap function and the quasiparticle coherence control the SC state. Therefore in our approach there is no need to introduce any phenomenological charge renormalization factors in order to describe the electromagnetic response $\mathrm{e}^{22}$.

Now we turn to the discussion of the electromagnetic response in the kinetic energy driven cuprate superconductors. The weak external magnetic field applied to the system usually represents a weak perturbation, but the induced field generated by supercurrents cancels this weak external field over most of the volume of the sample. Consequently, the net field acts only very near the surface on a scale of the magnetic field penetration depth and so it can be treated as a weak perturbation on the system as a whole. Therefore the electromagnetic response can be successfully studied within the linear response approach ${ }^{38.39}$, where the averaged value $\mathbf{J}$ of the induced microscopic screening current $\mathbf{j}$ in the presence of the vector potential $\mathbf{A}$ is found as,

$$
J_{\mu}(\mathbf{q}, \omega)=-\sum_{\nu=1}^{3} K_{\mu \nu}(\mathbf{q}, \omega) A_{\nu}(\mathbf{q}, \omega),
$$

where $K_{\mu \nu}$ is the kernel of the response function and the Greek indices label the axes of the Cartesian coordinate system. Recall that, as always in the linear response method, the thermal average of the supercurrent is calculated with the unperturbed Hamiltonian, i.e. for $\mathbf{A} \equiv 0$ in Eq. (2). Let us also note that the relation (4), which is local in the reciprocal space, in general implies a nonlocal response in the coordinate space.

The kernel, which plays a central role in the description of the electromagnetic response, and once known allows us to calculate quantitative characteristics of the electromagnetic response, can be separated into two parts:

$$
K_{\mu \nu}(\mathbf{q}, \omega)=K_{\mu \nu}^{(\mathrm{d})}(\mathbf{q}, \omega)+K_{\mu \nu}^{(\mathrm{p})}(\mathbf{q}, \omega),
$$


a diamagnetic part $K_{\mu \nu}^{(\mathrm{d})}$ and a paramagnetic one $K_{\mu \nu}^{(\mathrm{p})}$. The evaluation of the diamagnetic contribution usually poses no difficulties since it is known almost immediately from the form of the diamagnetic current operator: it turns out to be diagonal and proportional to the average kinetic term. However, the paramagnetic part can only be calculated approximately since it involves evaluation of a retarded current-current correlation function (polarization bubble). As the retarded function is inconvenient for perturbation analysis one usually proceeds with the corresponding imaginary-time-ordered Matsubara function,

$$
P_{\mu \nu}(\mathbf{q}, \tau)=-\left\langle T_{\tau}\left\{j_{\mu}^{(\mathrm{p})}(\mathbf{q}, \tau) j_{\nu}^{(\mathrm{p})}(-\mathbf{q}, 0)\right\}\right\rangle,
$$

where the paramagnetic current operator is defined in the imaginary time $\tau$ Heisenberg picture. Hence, the main problem is reduced to the evaluation of a retarded current commutator for the unperturbed system. The retarded current-current correlation function is then obtained in a standard way from the imaginary time Fourier transform $P_{\mu \nu}\left(\mathbf{q}, i \omega_{n}\right)$ of the Matsubara function (6) by analytic continuation to real frequencies 37 .

The vector potential A (then the weak external magnetic field $B=\operatorname{rot} \mathbf{A}$ ) has been coupled to the electrons, which are now represented by $C_{l \uparrow}=h_{l \uparrow}^{\dagger} S_{l}^{-}$and $C_{l \downarrow}=h_{l \downarrow}^{\dagger} S_{l}^{+}$in the CSS fermion-spin representation. However, in the CSS framework, the vector potential A is coupled to $h_{l \sigma}^{\dagger}$, while the corresponding weak external magnetic field $\mathbf{B}=\operatorname{rot} \mathbf{A}$ is coupled to $\mathbf{S}_{l}$ by including the Zeeman term ${ }^{40}$ in the Hamiltonian (1). For cuprate superconductors, the upper critical magnetic field is 50 Tesla or greater around the optimal doping. In this paper, we mainly focus on the case where the applied external magnetic field $B<10 \mathrm{mT}$ is much less than the upper critical magnetic field. In this case, the Zeeman term ${ }^{40}$ in the Hamiltonian (11) has been dropped, and then the electron current operator $j_{\mu}=j_{\mu}^{(\mathrm{d})}+j_{\mu}^{(\mathrm{p})}$ can be obtained by differentiating the Hamiltonian (2) with respect to the vector potential as,

$$
\begin{aligned}
& j_{\mu}^{(\mathrm{d})}=\frac{\chi e^{2} t}{2 \hbar^{2}} \sum_{l \sigma}\left(h_{l+\hat{\mu} \sigma}^{\dagger} h_{l \sigma}+h_{l \sigma}^{\dagger} h_{l+\hat{\mu} \sigma}\right) A_{\mu}(l), \\
& j_{\mu}^{(\mathrm{p})}=-\frac{i \chi e t}{2 \hbar} \sum_{l \sigma}\left(h_{l+\hat{\mu} \sigma}^{\dagger} h_{l \sigma}-h_{l \sigma}^{\dagger} h_{l+\hat{\mu} \sigma}\right),
\end{aligned}
$$

being the diamagnetic and paramagnetic contributions, respectively.

Since the diamagnetic current is explicitly proportional to the vector potential, it is straightforward to find the diamagnetic part of the response kernel as,

$$
\begin{aligned}
K_{\mu \nu}^{(\mathrm{d})}(\mathbf{q}) & =-\frac{Z_{\mathrm{hF}} \chi e^{2} t}{\hbar^{2}} \frac{1}{N} \sum_{\mathbf{k}} \delta_{\mu \nu} \cos k_{\mu} \\
& \times\left(1-\frac{\bar{\xi}_{\mathbf{k}}}{E_{\mathrm{hk}}} \tanh \frac{\beta E_{\mathrm{hk}}}{2}\right) \\
& =-\frac{2 \chi \phi e^{2} t}{\hbar^{2}} \delta_{\mu \nu} .
\end{aligned}
$$

The paramagnetic part of the response kernel is more complicated to calculate, as it involves evaluation of the current-current correlation function (6). In particular, if we want to keep the theory gauge invariant, it is crucial to approximate the correlation function in a way maintaining local charge conservation ${ }^{2,34,39,41}$. Since in the following calculations we will work with a fixed gauge of the vector potential, we postpone the detailed discussion of this problem until Appendix A Starting with the paramagnetic current operator (7b), we can rewrite its Fourier transform in the notation of Nambu fields $\Psi_{\mathbf{k}}^{\dagger}=\left(h_{\mathbf{k} \uparrow}^{\dagger}, h_{-\mathbf{k} \downarrow}\right)$ and $\Psi_{\mathbf{k}+\mathbf{q}}=\left(h_{\mathbf{k}+\mathbf{q} \uparrow}, h_{-\mathbf{k}-\mathbf{q} \downarrow}^{\dagger}\right)^{T}$ as

$j_{\mu}^{(\mathrm{p})}(\mathbf{q})=\frac{1}{N} \sum_{\mathbf{k}} \Psi_{\mathbf{k}}^{\dagger}\left[-\frac{\chi e t}{\hbar} e^{i \frac{q_{\mu}}{2}} \sin \left(k_{\mu}+\frac{q_{\mu}}{2}\right) \tau_{0}\right] \Psi_{\mathbf{k}+\mathbf{q}}$

For the purpose of the discussion addressing the gauge invariance problem, presented in Appendix $\mathrm{A}$, it is convenient to find the charge density in the Nambu notation as well. Within the CSS fermion-spin scheme, we first find $\rho(\mathbf{q}) \approx-(e / 2 N) \sum_{\mathbf{k}}\left(\delta_{\mathbf{q}, 0}-h_{\mathbf{k} \uparrow}^{\dagger} h_{\mathbf{k}+\mathbf{q} \uparrow}-\right.$ $\left.h_{\mathbf{k} \downarrow}^{\dagger} h_{\mathbf{k}+\mathbf{q} \downarrow}\right)$. Then the paramagnetic four-current operator can be represented in the Nambu form as $j_{\mu}^{(\mathrm{p})}(\mathbf{q})=$ $\sum_{\mathbf{k}} \Psi_{\mathbf{k}}^{\dagger} \gamma_{\mu}(\mathbf{k}+\mathbf{q}, \mathbf{k}) \Psi_{\mathbf{k}+\mathbf{q}}$, where the bare current vertex,

$$
\gamma_{\mu}(\mathbf{k}+\mathbf{q}, \mathbf{k})= \begin{cases}-\frac{\chi e t}{\hbar} e^{i \frac{q_{\mu}}{2}} \sin \left(k_{\mu}+\frac{q_{\mu}}{2}\right) \tau_{0} & \text { for } \mu \neq 0 \\ -\frac{e^{2}}{2} \tau_{3} & \text { for } \mu=0\end{cases}
$$

It is necessary to be aware that we are calculating the polarization bubble with the paramagnetic current operator (9), i.e., bare current vertices (10), but charge carrier Green functions. Consequently, as in this scenario we do not take into account longitudinal excitations properly ${ }^{2.34}$, the obtained results are valid only in the gauge, where the vector potential is purely transverse, e.g. in the Coulomb gauge. In this case, we can obtain the correlation function (6) in the Matsubara representation as,

$$
P_{\mu \nu}\left(\mathbf{q}, i \omega_{n}\right)=\left(\frac{\chi e t}{\hbar}\right)^{2} e^{\frac{i}{2}\left(q_{\mu}-q_{\nu}\right)} \frac{1}{N} \sum_{\mathbf{k}} \sin \left(k_{\mu}+\frac{q_{\mu}}{2}\right) \sin \left(k_{\nu}+\frac{q_{\nu}}{2}\right) \frac{1}{\beta} \sum_{i \nu_{m}} \operatorname{Tr}\left[\mathbb{G}\left(\mathbf{k}+\mathbf{q}, i \omega_{n}+i \nu_{m}\right) \mathbb{G}\left(\mathbf{k}, i \nu_{m}\right)\right]
$$


Restricting the discussion to the static limit $(\omega \sim 0)$ and completing the summation over Matsubara frequencies, we obtain the bare vertex current-current correla- tion function, and hence the paramagnetic part of the response kernel as,

$$
\begin{aligned}
K_{\mu \nu}^{(\mathrm{p})}(\mathbf{q}, 0) & =-\left(\frac{\chi e t Z_{\mathrm{hF}}}{\hbar^{2}}\right)^{2} e^{\frac{i}{2}\left(q_{\mu}-q_{\nu}\right)} \frac{1}{N} \sum_{\mathbf{k}} \sin \left(k_{\mu}+\frac{q_{\mu}}{2}\right) \sin \left(k_{\nu}+\frac{q_{\nu}}{2}\right) \\
& \times\left\{\frac{1}{E_{\mathrm{h} \mathbf{k}}+E_{\mathrm{h} \mathbf{k}+\mathbf{q}}}\left[1-\frac{\bar{\xi}_{\mathbf{k}+\mathbf{q} \bar{\xi}_{\mathbf{k}}}+\bar{\Delta}_{\mathrm{hZ}}(\mathbf{k}+\mathbf{q}) \bar{\Delta}_{\mathrm{hZ}}(\mathbf{k})}{E_{\mathrm{h} \mathbf{k}} E_{\mathrm{h} \mathbf{k}+\mathbf{q}}}\right]\left[1-n_{\mathrm{F}}\left(E_{\mathrm{h} \mathbf{k}}\right)-n_{\mathrm{F}}\left(E_{\mathrm{h} \mathbf{k}+\mathbf{q}}\right)\right]\right. \\
& \left.+\frac{1}{E_{\mathrm{h} \mathbf{k}}-E_{\mathrm{h} \mathbf{k}+\mathbf{q}}}\left[1+\frac{\bar{\xi}_{\mathbf{k}+\mathbf{q}} \bar{\xi}_{\mathbf{k}}+\bar{\Delta}_{\mathrm{hZ}}(\mathbf{k}+\mathbf{q}) \bar{\Delta}_{\mathrm{hZ}}(\mathbf{k})}{E_{\mathrm{h} \mathbf{k}} E_{\mathrm{h} \mathbf{k}+\mathbf{q}}}\right]\left[n_{\mathrm{F}}\left(E_{\mathrm{h} \mathbf{k}+\mathbf{q}}\right)-n_{\mathrm{F}}\left(E_{\mathrm{hk}}\right)\right]\right\}
\end{aligned}
$$

Note that in the long wavelength limit, when $|\mathbf{q}| \rightarrow 0$, the former term in Eq. (12) vanishes, and the latter turns into $-2\left(\chi e t Z_{\mathrm{hF}} / N \hbar^{2}\right)^{2} \sum_{\mathbf{k}} \sin k_{\mu} \sin k_{\nu} n_{\mathrm{F}}\left(E_{\mathrm{hk}}\right)[1-$ $\left.n_{\mathrm{F}}\left(E_{\mathrm{hk}}\right)\right]$, which is equal to zero in the zero-temperature limit. Hence, in this case, the long wavelength electromagnetic response at zero temperature is determined by the diamagnetic part of the kernel only.

\section{QUANTITATIVE CHARACTERISTICS}

The way the system reacts to a weak electromagnetic stimulus is entirely described by the linear response kernel, which is calculated within a microscopic model. Once the kernel is known, the effect of a weak external magnetic field can be quantitatively characterized by experimentally measurable quantities such as the magnetic field penetration depth and the local magnetic field profile. Technically, we need to combine one of the Maxwell equations with the relation (4) describing the response of the system and solve them together for the vector potential. This is the step in which a particular gauge of the vector potential-usually implied by the geometry of the system - is set. However, the kernel function derived within the linear response theory describes the response of an infinite system. In order to take into account the confined geometry of cuprate superconductors it is necessary to introduce a surface being the boundary between the environment and the sample. This can be done within the standard specular reflection model ${ }^{31,32}$ with a two-dimensional geometry of the SC plane, in the configuration with external magnetic field perpendicular to the ab plane, as shown in Fig. 1. In the present paper we study magnetic field penetration effects within the ab plane only, so our primary goal is to find and discuss the magnetic field in-plane penetration depth.

In order to simulate an external magnetic field at the surface of a two-dimensional sample, we introduce an external current sheet $J_{y, \text { ext }}(x)=-2 B_{0} \delta(x) / \mu_{0}$ at the edge $x=0$, where $\mu_{0}$ is the magnetic permeabil-

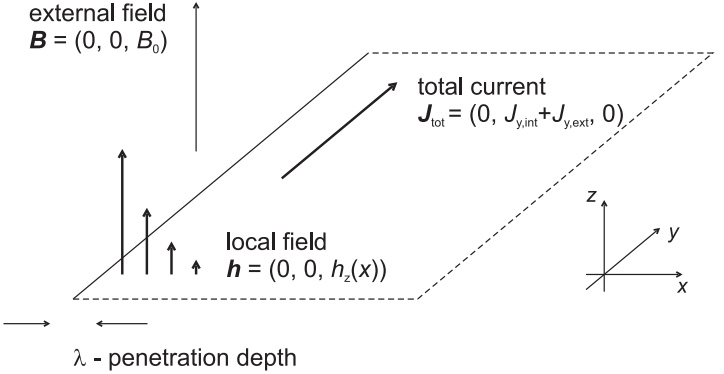

FIG. 1: Geometry of the specular reflection model. The current $\mathbf{J}_{\text {ext }}$ simulates external magnetic field at the edge of the sample $(x=0)$, whereas the induced supercurrent $\mathbf{J}_{\text {int }}$ is the (linear) reaction of the system.

ity and $B_{0}$ is the amplitude of the weak external magnetic field at the surface $(x=0)$. From the Maxwell equation for the curl of the local magnetic field $\operatorname{rot} \mathbf{h}=$ $\mu_{0}\left(\mathbf{J}_{\text {int }}+\mathbf{J}_{\text {ext }}\right)=\mu_{0} \mathbf{J}_{\text {int }}+\left[0,-2 B_{0} \delta(x), 0\right]$ and the fact, that the induced supercurrent $\mathbf{J}_{\text {int }}$ flows along the $y$ axis, we can state that the local magnetic field is of the form $\mathbf{h}(\mathbf{r})=\left[0,0, h_{z}(x)\right]$. In order to discuss the magnetic field penetration effect, spatial dependence of the local magnetic field has to be found. Let us begin with the identity $\operatorname{rot} \operatorname{rot} \mathbf{A}=\operatorname{grad} \operatorname{div} \mathbf{A}-\nabla^{2} \mathbf{A}$ and choose the vector potential as $\mathbf{A}(\mathbf{r})=\left[0, A_{y}(x), 0\right]$ setting the Coulomb gauge. In this case, $q_{x}^{2} A_{y}(\mathbf{q})=\mu_{0}\left[J_{y, \text { int }}(\mathbf{q})+J_{y, \text { ext }}(\mathbf{q})\right]$, because the vector potential has only non-zero $y$ component. Finally, including the form of the external current, the linear relation (4) between the induced supercurrent and the vector potential $J_{y \text {,int }}(\mathbf{q})=-K_{y y}(\mathbf{q}) A_{y}(\mathbf{q})$, and solving for the vector potential we obtain,

$$
A_{y}(\mathbf{q})=-8 \pi^{2} B_{0} \frac{\delta\left(q_{y}\right) \delta\left(q_{z}\right)}{\mu_{0} K_{y y}(\mathbf{q})+q_{x}^{2}} .
$$

Since the vector potential has only the $y$ component, the only non-zero component of the local magnetic field $\mathbf{h}=\operatorname{rot} \mathbf{A}$ is that along the $z$ axis and $h_{z}(\mathbf{q})=i q_{x} A_{y}(\mathbf{q})$. Substituting the derived form of the vector potential (13), and taking the inverse Fourier transform, the local mag- 
netic field profile can be obtained as,

$$
h_{z}(x)=\frac{B_{0}}{\pi} \int_{-\infty}^{\infty} \mathrm{d} q_{x} \frac{q_{x} \sin q_{x} x}{\mu_{0} K_{y y}\left(q_{x}, 0,0\right)+q_{x}^{2}} .
$$

Local magnetic field profiles can be measured experimentally, e.g. using the muon-spin rotation technique ${ }^{12.13}$, providing an important tool to investigate the details of magnetic field screening inside the sample. In cuprate superconductors the screening is found to be of exponential character ${ }^{12.13}$, in support of a local (London-type) nature of the electrodynamics ${ }^{2}$. For the convenience of the following discussions, we introduce a characteristic length scale $a_{0}=\sqrt{\hbar^{2} a / \mu_{0} e^{2} J}$. Using a reasonably estimative value of $J / k_{\mathrm{B}} \approx 1000 \mathrm{~K}$ and $a \approx 0.383 \mathrm{~nm}$, which is the lattice parameter for the cuprate superconductor $\mathrm{YBa}_{2} \mathrm{Cu}_{3} \mathrm{O}_{7-y}$, we obtain $a_{0} \approx 97.8 \mathrm{~nm}$. In Fig. 2, we plot the local magnetic field profile (14) as a function of the distance from the surface at temperature $T=0.02 J$ for the doping concentration $\delta=0.150$ (solid line), $\delta=0.147$ (dashed line), and $\delta=0.144$ (dotted line) with parameter $t=2.5 \mathrm{~J}$. For comparison, the corresponding experimental result $\frac{13}{13}$ of the local magnetic field profiles for the high quality $\mathrm{YBa}_{2} \mathrm{Cu}_{3} \mathrm{O}_{7-y}$ sample is also shown in Fig. 2 (inset, bottom). If a weak external field $B_{0} \approx 10 \mathrm{mT}$ is applied to the system just as it has been done in the experimental measurement 13 , then the experimental result $\frac{13}{3}$ for $\mathrm{YBa}_{2} \mathrm{Cu}_{3} \mathrm{O}_{7-y}$ is well reproduced. In particular, our theoretical results perfectly follow an exponential law as expected for the local electrodynamic response.

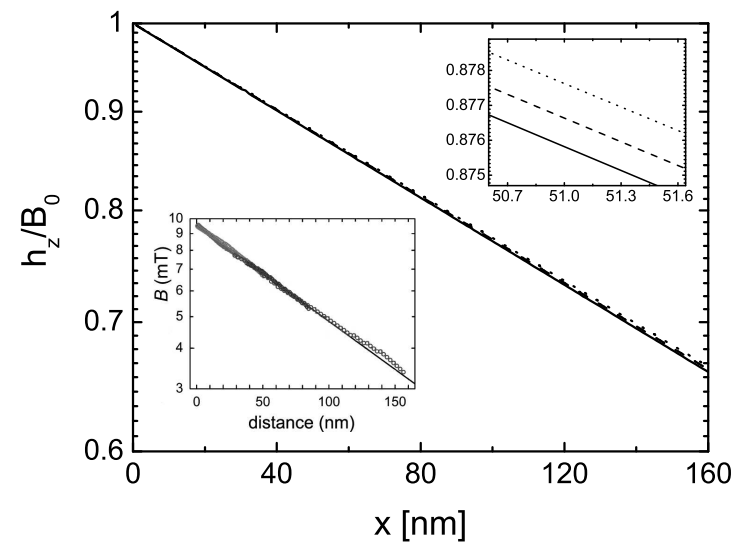

FIG. 2: The local magnetic field profile as a function of the distance from the surface at temperature $T=0.02 J$ for doping concentration $\delta=0.150$ (solid line), $\delta=0.147$ (dashed line), and $\delta=0.144$ (dotted line) with parameter $t=2.5 \mathrm{~J}$. Inset (top): zoom into the intermediate range of the local magnetic field profile. Inset (bottom): the corresponding experimental result for $\mathrm{YBa}_{2} \mathrm{Cu}_{3} \mathrm{O}_{7-y}$ taken from Ref. 13 .

The above obtained local magnetic field profile $h_{z}(x)$ allows us to determine the magnetic field in-plane penetration depth $\lambda(T)$ in a straightforward way. According

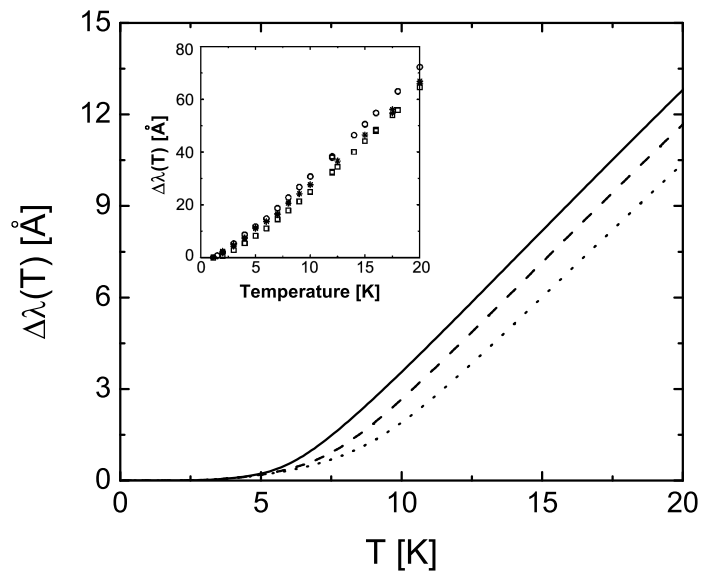

FIG. 3: Temperature dependence of the magnetic field inplane penetration depth $\Delta \lambda(T)$ for the doping concentration $\delta=0.150$ (solid line), $\delta=0.149$ (dashed line), and $\delta=0.148$ (dotted line) with parameter $t / J=2.5$. Inset: the corresponding experimental data for $\mathrm{YBa}_{2} \mathrm{Cu}_{3} \mathrm{O}_{7-y}$ taken from Ref. 8.

to the definition $\lambda(T)=B_{0}^{-1} \int_{0}^{\infty} h_{z}(x) \mathrm{d} x$, the magnetic field in-plane penetration depth can be evaluated as,

$$
\lambda(T)=\frac{2}{\pi} \int_{0}^{\infty} \frac{\mathrm{d} q_{x}}{\mu_{0} K_{y y}\left(q_{x}, 0,0\right)+q_{x}^{2}} .
$$

In this case, we obtain the zero-temperature magnetic field in-plane penetration depth $\lambda(0) \approx 380.8 \mathrm{~nm}$ for the doping concentration $\delta=0.150$ with parameter $t / J=2.5$. This anticipated value is very close to the values of the magnetic field in-plane penetration depth $\lambda \approx 156 \mathrm{~nm} \sim 400 \mathrm{~nm}$ observed in different families of cuprate superconductors $12,17,42$. Furthermore, $\Delta \lambda(T)=\lambda(T)-\lambda(0)$ as a function of temperature $T$ for the doping concentration $\delta=0.150$ (solid line), $\delta=0.149$ (dashed line), and $\delta=0.148$ (dotted line) with parameter $t / J=2.5$ is plotted in Fig. 3 in comparison with the corresponding experimental results $\frac{8}{\underline{-}}$ of $\mathrm{YBa}_{2} \mathrm{Cu}_{3} \mathrm{O}_{7-y}$ (inset). Our theoretical results show linear characteristics of the magnetic field in-plane penetration depth $\Delta \lambda(T)$, except for extremely low temperatures where a strong deviation from the linear characteristics (a nonlinear effect) appears. In particular, this crossover from the linear temperature dependence in the low temperature regime into the nonlinear one at extremely low temperatures is observed experimentally in nominally clean crystals of cuprate superconductors $3-8-14$. Apparently, there is a substantial difference between theory and experiment, namely, the value of the difference between $\lambda(T)$ and $\lambda(0)$ calculated theoretically is much smaller than the corresponding value measured in the experiment. However, upon a closer examination one can see immediately that the main difference is due to fact that the calculated $\lambda(T)$ increases slowly with temperature. As for a qualitative discussion in this paper, the overall tendency seen in the theoretical result is consistent with that in the 
experiment $\stackrel{8}{ }$. In cuprate superconductors, the values of $J$ and $t$ are believed to vary somewhat from compound to compound ${ }^{7}$. Therefore the quantitative agreement can be reached by adjustments of theory's parameters $t$ and $J$, or by introducing the next neighbor hopping $t^{\prime}$.

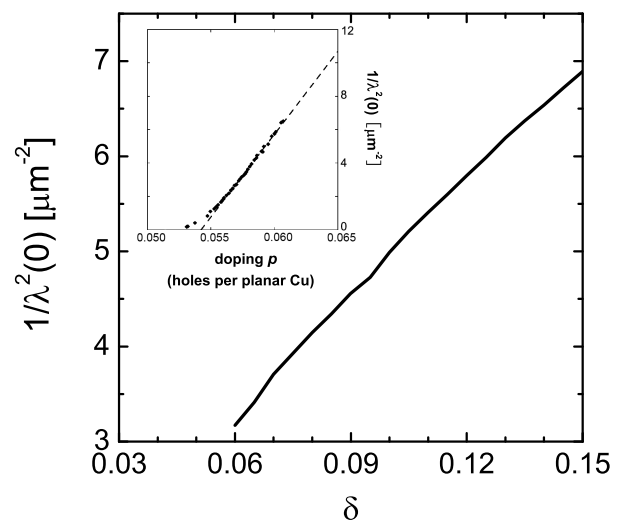

FIG. 4: Doping dependence of the zero-temperature inplane superfluid density in the underdoped regime with $t / J=2.5$. Inset: the corresponding experimental result for $\mathrm{YBa}_{2} \mathrm{Cu}_{3} \mathrm{O}_{7-y}$ taken from Ref. 16 .

A quantity which is closely related to the magnetic field in-plane penetration depth $\lambda(T)$ is the in-plane superfluid density $\rho_{\mathrm{s}}(T) \equiv \lambda^{-2}(T)$. For a better understanding of the physical properties of cuprate superconductors, we have calculated the doping dependence of the zero-temperature in-plane superfluid density $\rho_{\mathrm{s}}(0)$ in the underdoped regime. The result for parameter $t / J=2.5$ is plotted in Fig. 4in comparison with the corresponding experimental data 16 for $\mathrm{YBa}_{2} \mathrm{Cu}_{3} \mathrm{O}_{7-y}$ (inset). It is shown that the in-plane superfluid density $\rho_{\mathrm{s}}(0)$ in the underdoped regime vanishes more or less linearly with decreasing doping concentration $\delta$, in qualitative agreement with experimental results of cuprate superconductors $15-17$. This result also is a natural consequence of the linear doping dependence of the SC transition temperature $T_{c} \propto \delta$ in the underdoped regime in the framework of the kinetic energy driven SC mechanism 24 , where the SC transition temperature $T_{c}$ is set by the charge carrier doping concentration, and then the density of the charge carriers directly determines the in-plane superfluid density in the underdoped regime.

The appearance of the nonlinearity in the temperature dependence of the magnetic field in-plane penetration depth in cuprate superconductors at extremely low temperatures, as shown in Fig. 3, can be attributed to the nonlocal effects, which in the case of a pure d-wave cuprate superconductor with nodes in the gap become significant for the electromagnetic response $\underline{18-22}$. In general, the relation between the supercurrent and the vector potential (44) is nonlocal in the coordinate space due to the finite size of charge carrier Cooper pairs. In the framework of the kinetic energy driven d-wave SC mechanism, the size of charge carrier pairs in the clean limit is of the order of the coherence length $\zeta(\mathbf{k})=\hbar v_{\mathrm{F}} / \pi \Delta_{\mathrm{h}}(\mathbf{k})$, where $v_{\mathrm{F}}=\hbar^{-1} \partial \xi_{\mathbf{k}} /\left.\partial \mathbf{k}\right|_{k_{F}}$ is the charge carrier velocity at the Fermi surface, and therefore the size of charge carrier pairs is momentum dependent. Although the weak external magnetic field decays exponentially on the scale of the magnetic field in-plane penetration length $\lambda(T)$, any nonlocal contributions to measurable quantities are of the order of $\kappa^{-2}$, where $\kappa$, known as the GinzburgLandau parameter, is the ratio of the magnetic field in-plane penetration depth $\lambda$ and the coherence length $\zeta$. However, in the d-wave cuprate superconductors, the characteristic feature is the existence of four nodal points $[ \pm \pi / 2, \pm \pi / 2]$ in the Brillouin zone, where the charge carrier gap function vanishes $\left.\Delta_{\mathrm{h}}(\mathbf{k})\right|_{[ \pm \pi / 2, \pm \pi / 2]}=$ $\Delta_{\mathrm{h}}\left(\cos k_{x}-\cos k_{y}\right) /\left.2\right|_{[ \pm \pi / 2, \pm \pi / 2]}=0$. As a consequence, the coherence length $\zeta(\mathbf{k})$ diverges around the nodes. In particular, at extremely low temperatures, the quasiparticles selectively populate the nodal region, and the major contribution to measurable quantities comes from these quasiparticles. In this case, the Ginzburg-Landau ratio $\kappa(\mathbf{k})$ around the nodal region is no longer large enough for the system to belong to the class of typeII superconductors, and the condition of the local limit is not fulfilled 19 . On contrary, the system falls then into the extreme nonlocal limit, and therefore the nonlinear characteristic in the temperature dependence of the magnetic field in-plane penetration depth can be observed experimentally in cuprate superconductors at sufficiently low temperatures $3,12-14$. However, with increasing temperature, the quasiparticles around the nodal region become excited out of the condensate, and the nonlocal effect fades away. In this case, the momentum dependent coherence length $\zeta(\mathbf{k})$ can be replaced approximately with the isotropic one $\zeta_{0}=\hbar v_{\mathrm{F}} / \pi \Delta_{\mathrm{h}}$. Then the Ginzburg-Landau parameter $\kappa_{0} \approx \lambda(0) / \zeta_{0} \approx 180$, and the condition for the local limit is satisfied. This anticipated value of the Ginzburg-Landau parameter $\kappa_{0} \approx 180$ is not too far from the range $\kappa_{0} \approx 150 \sim 400$ estimated experimentally for different families of cuprate superconductors ${ }^{12,17,42}$. Consequently, the cuprate superconductors at moderately low temperatures turn out to be type-II superconductors, where nonlocal effects are negligible, the electrodynamics is purely local and the magnetic field decays exponentially over a length of the order of a few hundreds nm. In this local limit, the pure d-wave pairing state in the kinetic energy driven $\mathrm{SC}$ mechanism gives the magnetic field penetration depth $\lambda(T) \propto T^{4,19}$. This is why the linear temperature dependence of the magnetic field in-plane penetration depth $\lambda(T)$ is observed experimentally $\underline{3,5,8-11}$ in cuprate superconductors at moderately low temperatures.

Finally, we have to note that a deviation from the linear Uemura relation between the in-plane superfluid density $\rho_{\mathrm{s}}(0)$ and doping concentration $\delta$ has been observed recently in the underdoped regime ${ }^{11,16,43}$. This deviation from the linear Uemura relation suggests a sublinear dependence of the critical temperature $T_{\mathrm{c}}$ and the superfluid density $\rho_{\mathrm{s}}(0)$, since $T_{\mathrm{c}}$ must fall to zero when $\rho_{\mathrm{s}}(0)$ does 11,43 . The parent compound of doped cuprate super- 
conductors is a Mott insulator with an antiferromagnetic long-range order and superconductivity occurs when the antiferromagnetic long-range order state is suppressed by doped charge carriers. Since these doped charge carriers in cuprate superconductors are induced by the replacement of some ions by other ones with different valences, or the addition of excess oxygens in the block layer, therefore, in principle, all cuprate superconductors have natural impurities ${ }^{7}$. Therefore the impurities play an important role in the electromagnetic response and lead to some subtle differences in the electromagnetic response for different families of cuprate superconductors ${ }^{3}$. In this case, the impurity effect on the SC state of cuprate superconductors is also a possible source for the deviation from the linear Uemura relation. In this context we $\underline{\underline{44}}$ have discussed the effect of the extended impurity scatterers on the quasiparticle transport of cuprate superconductors in the SC state based on the nodal approximation of the quasiparticle excitations and scattering processes, and predicted that in contrast with the dome shape of the doping dependent SC gap parameter, the minimum of the microwave conductivity occurs around the optimal doping, and then increases in both underdoped and overdoped regimes. However, in this paper we are primarily interested in exploring the general notion of the electromagnetic response in cuprate superconductors in the SC state. The qualitative agreement between the present theoretical results in the clean limit and experimental data for different families of cuprate superconductors provides an important confirmation of the nature of the SC phase of cuprate superconductors as a d-wave BCS-like SC state within the kinetic energy driven SC mechanism.

\section{CONCLUSIONS}

In this paper we have discussed the electromagnetic response in cuprate superconductors within the framework of kinetic energy driven d-wave superconductivity. Following the linear response theory and taking into account the two-dimensional geometry of cuprate superconductors within the specular reflection model, we have reproduced some main features of the electromagnetic response experiments on cuprate superconductors, including the exponential local magnetic field profile, the linear temperature dependence of the in-plane penetration depth in the low temperature range and its nonlinear temperature dependence at extremely low temperatures. Moreover, the linear doping dependence of the zero-temperature in-plane superfluid density in the underdoped regime has been reproduced. In particular, we have clearly identified the limitations of the used approximations, especially with respect to the problem of gauge invariance. Furthermore, we have proposed a method to generalize the discussions in order to make them independent of a particular choice of the vector potential.

\section{Acknowledgments}

The authors would like to thank Dr. Zhi Wang and Dr. Yu Lan for helpful discussions. This work was supported by the National Natural Science Foundation of China under Grant No. 10774015, and the funds from the Ministry of Science and Technology of China under Grant Nos. 2006CB601002 and 2006CB921300. MK gratefully acknowledges support from a research scholarship funded by Institute of Physics, Wrocław University of Technology.

\section{Appendix A: Gauge-invariant electromagnetic response}

It is well known that gauge invariance is a direct consequence of local charge conservation ${ }^{2.39}$, which is mathematically expressed by the charge density-current continuity equation or its Green function analogue called the generalized Ward identity $(\mathrm{GWI})^{2,34,39,41}$

$$
-2 N \sum_{\mu=0}^{3} q_{\mu} \Gamma_{\mu}(k+q, k)=\tau_{3} \mathbb{G}^{-1}(k)-\mathbb{G}^{-1}(k+q) \tau_{3} .
$$

Here $\Gamma_{\mu}$ is a dressed version of the four-current vertex function, and the four-vector notation $q=\left(\mathbf{q}, q_{0}=i \omega\right)$ along with the metric $(1,1,1,-1)$ has been introduced.

Since the local charge conservation requirement is quite universal and fundamental, it should be inherent to any theory of the electromagnetic response which is expected to be gauge invariant. The purpose of this appendix is to propose - within the framework of the kinetic energy driven superconductivity - a method to dress the current vertex in a way, which does not violate the GWI. Once such a method is found, the bare polarization bubble (6) can be replaced with its dressed version presented in Fig. 5, and the resulting kernel of the response function will provide correct results for any gauge of the vector potential.

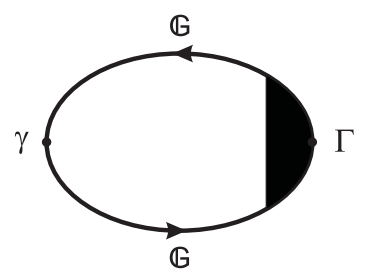

FIG. 5: Dressed polarization bubble (Nambu notation). Here both the Green function and the current vertex are dressed with the pairing interaction due to the spin bubble.

In the first step we will note that

$-2 N \sum_{\mu=0}^{3} q_{\mu} \gamma_{\mu}(k+q, k)=\tau_{3} \mathbb{G}^{(0)-1}(k)-\mathbb{G}^{(0)-1}(k+q) \tau_{3}$, 
i.e. that the GWI for the bare current vertex is satisfied with the MF charge carrier Green function $\mathbb{G}^{(0)}(k)=\left[\left(i \omega_{n}\right)^{2}-\xi_{\mathbf{k}}^{2}\right]^{-1}\left(i \omega_{n} \tau_{0}+\xi_{\mathbf{k}} \tau_{3}\right)$. Substituting the MF charge carrier Green function, the rhs of Eq. A2 turns into $\tau_{3} \mathbb{G}^{(0)-1}(k)-\mathbb{G}^{(0)-1}(k+q) \tau_{3}=$ $\left(\xi_{\mathbf{k}+\mathbf{q}}-\xi_{\mathbf{k}}\right) \tau_{0}-q_{0} \tau_{3}$. Moreover, in the long wavelength limit, after including the explicit form of the MF charge carrier dispersion relation found within the framework of the kinetic energy driven superconductivity ${ }^{26}$, it further simplifies to $\tau_{3} \mathbb{G}^{(0)-1}(k)-\mathbb{G}^{(0)-1}(k+q) \tau_{3} \approx$ $\left[-2 t \chi\left(q_{x} \sin k_{x}+q_{y} \sin k_{y}\right)\right] \tau_{0}-q_{0} \tau_{3}$. Now, recalling the form of the bare vertex (10), we can notice that in the long wavelength limit the scalar product on the left-hand side of Eq. A2 $-q_{0} \gamma_{0}+\mathbf{q} \gamma=$ $(2 N)^{-1}\left(\tau_{3} q_{0}-\tau_{0} \nabla_{\mathbf{k}} \xi_{\mathbf{k}} \cdot \mathbf{q}\right)$, which proves the equality (A2).

It is well known that in order to obtain a dressed vertex function, which does not violate the GWI, a ladder-type approximation can be adapted ${ }^{2,34,39}$. The nature of the pairing mechanism ${ }^{24,26}$, which originates from the spin bubble, suggests a ladder-like approximation of the form,

$$
\begin{aligned}
\Gamma_{\mu}(k+q, k) & =\gamma_{\mu}(k+q, k)+\frac{1}{N} \frac{1}{\beta} \sum_{p} \tau_{3} \mathbb{G}(k+p+q) \Gamma_{\mu}(k+p+q, k+p) \mathbb{G}(k+p) \tau_{3} \\
& \times \frac{1}{N} \sum_{\mathbf{p}^{\prime}} \Lambda_{\mathbf{p}+\mathbf{p}^{\prime}+\mathbf{k}}^{2} \Pi\left(\mathbf{p}, \mathbf{p}^{\prime} ; i p_{m}\right),
\end{aligned}
$$

which is graphically presented in Fig. 6.

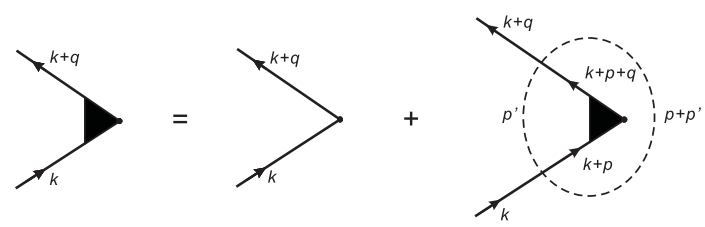

FIG. 6: Ladder-type approximation for the dressed vertex.
In order to prove that the approximation A3 for the dressed vertex in fact implies a gauge invariant description of the electromagnetic response, it is necessary and sufficient to check whether it does not violate the GWI A1 . In order to prove it, we insert the dressed vertex function (A3) into the left-hand side of Eq. (A1) and use the identity $-2 N \sum_{\mu=0}^{3} q_{\mu} \Gamma_{\mu}(s+q, s)=$ $\tau_{3} \mathbb{G}^{-1}(s)-\mathbb{G}^{-1}(s+q) \tau_{3}$ to obtain

$$
\begin{aligned}
\sum_{\mu=0}^{3} q_{\mu} \Gamma_{\mu}(k+q, k) & =\sum_{\mu=0}^{3} q_{\mu} \gamma_{\mu}(k+q, k)+\frac{1}{N} \frac{1}{\beta} \sum_{p}\left(-\frac{1}{2 N}\right)\left[\tau_{3} \mathbb{G}(k+p+q) \tau_{3}-\tau_{3} \mathbb{G}(k+p) \tau_{3}\right] \\
& \times \frac{1}{N} \sum_{\mathbf{p}^{\prime}} \Lambda_{\mathbf{p}+\mathbf{p}^{\prime}+\mathbf{k}}^{2} \Pi\left(\mathbf{p}, \mathbf{p}^{\prime} ; i p_{m}\right)
\end{aligned}
$$

In the long wavelength limit we use the approximation $\Lambda_{\mathbf{p}+\mathbf{p}^{\prime}+\mathbf{k}}^{2} \approx \Lambda_{\mathbf{p}+\mathbf{p}^{\prime}+\mathbf{k}+\mathbf{q}}^{2}$. Then we can simplify Eq. (A4) into $\sum_{\mu=0}^{3} q_{\mu} \Gamma_{\mu}(k+q, k) \approx \sum_{\mu=0}^{3} q_{\mu} \gamma_{\mu}(k+q, k)-$ $\left[\Sigma(k+q) \tau_{3}-\tau_{3} \Sigma(k)\right] / 2 N$. Using the fact that the free vertex satisfies the GWI with the MF Green function, as stated in Eq. (A2), and arranging the terms with respect to the Pauli matrices, we have

$$
\begin{aligned}
-2 N \sum_{\mu=0}^{3} q_{\mu} \Gamma_{\mu}(k+q, k) & \approx \tau_{3}\left[\mathbb{G}^{(0)-1}(k)-\Sigma(k)\right] \\
& -\left[\mathbb{G}^{(0)-1}(k+q)-\Sigma(k+q)\right] \tau_{3} .
\end{aligned}
$$

Hence, identifying the terms in the square brackets as dressed charge carrier Green functions, we eventually obtain the GWI (A1), what proves that the ladder-type approximation (A3) for the vertex function in the dressed polarization bubble in Fig. 5 is consistent with the GWI. Consequently, the kernel of the linear response calculated with the dressed polarization bubble is gauge invariant. 
* To whom correspondence should be addressed, E-mail: spfeng@bnu.edu.cn

2 See, e.g., J.R. Schrieffer, Theory of Superconductivity (Addison-Wesley, San Francisco, 1964).

3 See, e.g., B. A. Bonn and W. N. Hardy, in Physical Properties of High Temperature Superconductors V, edited by D.M. Ginsberg (World Scientific, Singapore, 1996).

4 See, e.g., C.C. Tsuei and J.R. Kirtley, Rev. Mod. Phys. 72 (2000) 969, and references therein.

5 W.N. Hardy, D.A. Bonn, D.C. Morgan, Ruixing Liang, and Kuan Zhang, Phys. Rev. Lett. 70 (1993) 3999.

6 H. Ding, J.C. Campuzano, K. Gofron, C. Gu, R. Liu, B. W. Veal, G. Jennings, Phys. Rev. B. 50 (1994) 1333; H. Ding, J.C. Campuzano, A.F. Bellman, T. Yokoya, M.R. Norman, M. Randeria, T. Takahashi, H. Katayama-Yoshida, T. Mochiku, K. Kadowaki, and G. Jennings, Phys. Rev. Lett. 74 (1995) 2784.

7 See, e.g., A. Damascelli, Z. Hussain, and Z.X. Shen, Rev. Mod. Phys. 75 (2003) 473.

8 S. Kamal, Ruixing Liang, A. Hosseini, D.A. Bonn, and W.N. Hardy, Phys. Rev. B. 58 (1998) R8933.

9 T. J. Jackson, T. M. Riseman, E. M. Forgan, H. Glückler, T. Prokscha, E. Morenzoni, M. Pleines, Ch. Niedermayer, G. Schatz, H. Luetkens, and J. Litterst, Phys. Rev. Lett. 84 (2000) 4958; Kuan Zhang, D. A. Bonn, S. Kamal, Ruixing Liang, D. J. Baar, W. N. Hardy, D. Basov, and T. Timusk, Phys. Rev. Lett. 73 (1994) 2484; Jian Mao, D. H. Wu, J. L. Peng, R. L. Greene, and Steven M. Anlage, Phys. Rev. B 51 (1995) 3316.

10 C. Panagopoulos, B. D. Rainford, J. R. Cooper, W. Lo, J. L. Tallon, J. W. Loram, J. Betouras, Y. S. Wang, and C. W. Chu, Phys. Rev. B. 60 (1999) 14617; T. Jacobs, S. Sridhar, Qiang Li, G. D. Gu, and N. Koshizuka, Phys. Rev. Lett. 75 (1995) 4516; Shih-Fu Lee, D. C. Morgan, R. J. Ormeno, D. M. Broun, R. A. Doyle, J. R. Waldram, and K. Kadowaki, Phys. Rev. Lett. 77 (1996) 735.

11 T. Pereg-Barnea, P.J. Turner, R. Harris, G.K. Mullins, J.S. Bobowski, M. Raudsepp, Ruixing Liang, D.A. Bonn, and W.N. Hardy, Phys. Rev. B 69 (2004) 184513; C. Panagopoulos, J. R. Cooper, G. B. Peacock, I. Gameson, P. P. Edwards, W. Schmidbauer, and J. W. Hodby, Phys. Rev. B 53 (1996) R2999.

12 R. Khasanov, D.G. Eshchenko, H. Luetkens, E. Morenzoni, T. Prokscha, A. Suter, N. Garifianov, M. Mali, J. Roos, K. Conder, and H. Keller, Phys. Rev. Lett. 92 (2004) 057602.

13 A. Suter, E. Morenzoni R. Khasanov, H. Luetkens, T. Prokscha, and N. Garifianov, Phys. Rev. Lett. 92 (2004) 087001.

14 J.E. Sonier, J.H. Brewer, R.F. Kiefl, G.D. Morris, R. Miller, D.A. Bonn, J. Chakhalian, R.H. Heffner, W.N. Hardy, and R. Liang, Phys. Rev. Lett. 83 (1999) 4156; M.H.S. Amin, M. Franz, and I. Affleck, Phys. Rev. Lett. 84 (2000) 5864.

15 Y. Uemura, G. M. Luke, B. J. Sternlieb, J. H. Brewer, J. F. Carolan, W. N. Hardy, R. Kadono, J. R. Kempton, R. F. Kiefl, S. R. Kreitzman, P. Mulhern, T. M. Riseman, D. Ll. Williams, B. X. Yang, S. Uchida, H. Takagi, J. Gopalakrishnan, A. W. Sleight, M. A. Subramanian, C. L. Chien, M. Z. Cieplak, Gang Xiao, V. Y. Lee, B. W. Statt, C. E. Stronach, W. J. Kossler, and X. H. Yu, Phys. Rev. Lett. 62 (1989) 2317; Y. J. Uemura, L. P. Le, G. M. Luke, B.
J. Sternlieb, W. D. Wu, J. H. Brewer, T. M. Riseman, C. L. Seaman, M. B. Maple, M. Ishikawa, D. G. Hinks, J. D. Jorgensen, G. Saito, and H. Yamochi, Phys. Rev. Lett. 66 (1991) 2665.

16 D. M. Broun, W. A. Huttema, P. J. Turner, S. Özcan, B. Morgan, Ruixing Liang, W. N. Hardy, and D. A. Bonn, Phys. Rev. Lett. 99 (2007) 237003.

17 C. Bernhard, J.L. Tallon, Th. Blasius, A. Golnik, and Ch. Niedermeyer, Phys. Rev. Lett. 86 (2001) 1614.

18 S.K. Yip and J. Sauls, Phys. Rev. Lett. 69 (1992) 2264.

19 I. Kosztin and A.J. Legget, Phys. Rev. Lett. 79 (1997) 135.

20 M. Franz, I. Affleck, and M. H. S. Amin, Phys. Rev. Lett. 79 (1997) 1555.

21 Mei-Rong Li, P.J. Hirschfeld, and P. Wölfle, Phys. Rev. B 61 (2000) 648.

22 D.E. Sheehy, T.P. Davis, and M. Franz, Phys. Rev. B 70 (2004) 054510.

${ }^{23}$ H. Matsui, T. Sato, T. Takahashi, S.C. Wang, H.B. Yang, H. Ding, T. Fujii, T. Watanabe, and A. Matsuda, Phys. Rev. Lett. 90 (2003) 217002; J. C. Campuzano, H. Ding, M. R. Norman, M. Randeira, A. F. Bellman, T. Yokoya, T. Takahashi, H. Katayama-Yoshida, T. Mochiku, and K. Kadowaki Phys. Rev. B 53 (1996) R14737.

24 Shiping Feng, Phys. Rev. B 68 (2003) 184501; Shiping Feng, Tianxing Ma, and Huaiming Guo, Physica C 436 (2006) 14.

25 P.W. Anderson, in: Frontiers and Borderlines in Many Particle Physics, edited by R. A. Broglia and J.R. Schrieffer (North-Holland, Amsterdam, 1987), p. 1; Science 235 (1987) 1196.

26 See, e.g., the review: Shiping Feng, Huaiming Guo, Yu Lan, and Li Cheng, Int. J. Mod. Phys. B 22 (2008) 3757, and references therein.

27 Huaiming Guo and Shiping Feng, Phys. Lett. A 361 (2007) 382; Shiping Feng and Tianxing Ma, Phys. Lett. A 350 (2006) 138; Yu Lan, Jihong Qin, and Shiping Feng, Phys. Rev. B 76 (2007) 014533; Weifang Wang, Zhi Wang, Jingge Zhang, and Shiping Feng, Phys. Lett. A 374 (2010) 632.

$28 \mathrm{Li}$ Cheng and Shiping Feng, Phys. Rev. B 77 (2008) 054518; Shiping Feng, Tianxing Ma, and Xintian Wu, Phys. Lett. A 352 (2006) 438; Shiping Feng and Tianxing Ma, in Superconductivity Research Horizons, edited by Eugene H. Peterson (Nova Science Publishers, Nrw York, 2007) chapter 5, pp. 129.

29 P. Dai, H.A. Mook, R.D. Hunt, and F. Dog̃an, Phys. Rev. B 63 (2001) 054525; Ph. Bourges, B. Keimer, S. Pailhés, L.P. Regnault, Y. Sidis, and C. Ulrich, Physica C 424 (2005) 45.

30 M. Arai, T. Nishijima, Y. Endoh, T. Egami, S. Tajima, K. Tomimoto, Y. Shiohara, M. Takahashi, A. Garret, and S.M. Bennington, Phys. Rev. Lett. 83 (1999) 608; S.M. Hayden, H.A. Mook, P. Dai, T.G. Perring, and F. Dog̃an, Nature 429 (2004) 531; C. Stock, W.J.L. Buyers, R.A. Cowley, P.S. Clegg, R. Coldea, C.D. Frost, R. Liang, D. Peets, D. Bonn, W.N. Hardy, and R.J. Birgeneau, Phys. Rev. B 71 (2005) 024522.

31 See, e.g., L.D. Landau, L.P. Pitaevskii, Statistical Physics (Part II) (Pergamon Press Ltd., 1980) Sec. 52.

32 See, e.g., M. Tinkham, Introduction to Superconductivity (McGraw-Hill, 1996) Appendix 3.

33 J.E. Hirsch and F. Marsiglio, Phys. Rev. B 45 (1992) 4807; 
D.J. Scalapino, S.R. White, and S. C. Zhang, Phys. Rev. Lett. 68 (1992) 2830; Phys. Rev. B 47 (1993) 7995; T. Kostyrko, R. Micnas, and K.A. Chao, Phys. Rev. B 49 (1994) 6158.

34 S. Misawa, Phys. Rev. B 49 (1994) 6305.

35 Shiping Feng, Jihong Qin, and Tianxing Ma, J. Phys.: Condens. Matter 16 (2004) 343; Shiping Feng, Tianxing Ma, and Jihong Qin, Mod. Phys. Lett. B 17 (2003) 361.

36 R.B. Laughlin, Phys. Rev. Lett. 79 (1997) 1726; J. Low. Tem. Phys. 99 (1995) 443.

37 See, e.g., G.D. Mahan, Many-Particle Physics, (Plenum Press, New York, 1981); G.M. Eliashberg, Sov. Phys. JETP 11 (1960) 696; D.J. Scalapino, J.R. Schrieffer, and J.W. Wilkins, Phys. Rev. 148 (1966) 263.

38 See, e.g., A.L. Fetter and J.D. Walecka, Quantum Theory of Many-Particle Systems (McGraw-Hill, 1971) Sec. 13.52.

39 H. Fukuyama, H. Ebisawa, and Y. Wada, Prog. Theor. Phys. 42 (1969) 494; H. Fukuyama, Prog. Theor. Phys. 42 (1969) 1284.

40 Jingge Zhang, Li Cheng, Huaiming Guo, and Shiping Feng, J. Magn. Magn. Mater. 321 (2009) 216.

41 P.I. Arseev, S.O. Loiko, N.K. Fedorov, Phys.-Usp. 49 (2006) 1.
${ }^{42}$ Y. J. Uemura, A. Keren, L. P. Le, G. M. Luke, W. D. Wu, Y. Kubo, T. Manako, Y. Shimakawa, M. Subramanian, J. L. Cobb, and J. T. Markert, Nature 364 (1993) 605; Ch. Niedermayer, C. Bernhard, U. Binninger, H. Gückler, J. L. Tallon, E. J. Ansaldo, and J. I. Budnick, Phys. Rev. Lett. 71 (1993) 1764; M. Nideröst, R. Frassanito, M. Saalfrank, A. C. Mota, G. Blatter, V. N. Zavaritsky, T. W. Li, and P. H. Kes, Phys. Rev. Lett. 81 (1998) 3231; S. L. Lee, P. Zimmermann, H. Keller, M. Warden, I. M. Savić, R. Schauwecker, D. Zech, R. Cubitt, E. M. Forgan, P. H. Kes, T. W. Li, A. A. Menovsky, and Z. Tarnawski, Phys. Rev. Lett. 71 (1993) 3862; C. Panagopoulos, B. D. Rainford, J. R. Cooper, W. Lo, J. L. Tallon, J. W. Loram, J. Betouras, Y. S. Wang and C. W. Chu, Phys. Rev. B 60 (1999) 14617; L. Fábrega, A. Calleja, A. Sin, S. Piñol, X. Obradors, J. Fontcuberta, and P. J. C. King, Phys. Rev. B 60 (1999) 7579 .

43 J. L. Tallon, J. W. Loram, J. R. Cooper, C. Panagopoulos, and C. Bernhard, Phys. Rev. B 68 (2003) 180501.

44 Zhi Wang, Huaiming Guo, and Shiping Feng, Physica C 468 (2008) 1078; Zhi Wang and Shiping Feng, Phys. Rev. B 80 (2009) 174507. 